\title{
Ischaemia in the Zinn-Haller circle and glaucomatous optic neuropathy in macaque monkeys
}

\author{
Mari Hiraoka, ${ }^{1}$ Kenichi Inoue, ${ }^{2}$ Taihei Ninomiya, ${ }^{2}$ Masahiko Takada ${ }^{2}$
}

${ }^{1}$ Division of Brain Development and Neuroregeneration, Tokyo Metropolitan Institute of Medical Science, Setagaya-ku, Tokyo, Japan

${ }^{2}$ Systems Neuroscience Section, Primate Research Institute, Kyoto University, Inuyama, Aichi, Japan

\section{Correspondence to} Dr Mari Hiraoka, Eye Research Division, Central Eye Infirmary, 1-24-15 Nukui-kitamachi, Koganei, Tokyo 184-0015, Japan;

mari9190@true.ocn.ne.jp

Accepted 8 November 2011

Published Online First

4 January 2012

\section{ABSTRACT}

Aims To elucidate the morphological features of optic neuropathy in an ischaemic model of glaucoma in macaque monkeys.

Methods The regional degenerative process was investigated by experimentally occluding the paraoptic branches of the lateral short posterior ciliary artery, that is, the circle of Haller and Zinn, in 11 eyes. Morphological changes in nerve fibres in the lamina cribrosa were evaluated by histopathology, immunocytochemistry and angiography, and the findings were compared with those observed in an aged macaque with spontaneous glaucomatous optic neuropathy.

Results Retinal ganglion cell axons were grouped in bundles and traversed through pores in columns of the lamina cribrosa. The processes of astrocytes extended to the bundles, and capillaries branched in surrounding connective tissue from the circular arterioles.

Experimental ischaemia induced time-dependent anoxic deterioration of phosphorylated fibres in the temporal arcuate zone, accompanied by glial proliferation. A monkey with spontaneous visual impairment had nerve fibre loss and gliosis with collagenous proliferation in the temporal hemisphere, suggesting glaucomatous neuropathy.

Conclusions Circulatory interference in the circle of Haller and Zinn caused time-dependent deterioration in the area where anoxic segmental degeneration is associated with pathogenesis of open-angle glaucoma.

\section{INTRODUCTION}

The peripapillary artery, also known as the circle of Haller and Zinn, is the vessel that provides most of the blood supply to the lamina cribrosa (LC) region of the optic nerve. Over 100 million retinal ganglion cell (RGC) axons group together into approximately 1 million axon fascicles, ${ }^{1}$ which form bundles of various sizes and traverse through the laminar pores to the neurons of the lateral geniculate nucleus (LGN). At all points, the biomechanical properties of this long pathway are subject to nerve damage. Mechanical injury to the axon causes astrocyte reactivation and subsequent remodelling of the extracellular matrix (ECM) in the LC. ${ }^{2} 3$ Furthermore, vascular nutrition in the axon is vital. The circle of Haller and Zinn comprises complete or incomplete anastomoses around the optic nerve between the medial and lateral short posterior ciliary arteries (SPCAs), which form a dense capillary plexus around the optic nerve. However, there is controversy regarding the distributary variation and characteristics of these anastomoses. Corrosion cast studies in humans and primates revealed a circular anastomosis, which allows circulatory compensation to circumvent segmental deficits. ${ }^{4-7}$
By contrast, angiography showed segmental filling that is supplied by an end artery. ${ }^{8} 9$ Furthermore, surgical intervention in the lateral SPCA induced interruption of fast axonal transport and a segmental filling defect in the focal blood supply. ${ }^{10} 11$ To determine whether damage to the circle of Haller and Zinn causes a segmental insult depending on end-arterial supply or diffuse deterioration due to compensatory coverage, the present study used moderate mechanical occlusion of the paraoptic branch of the lateral SPCA to induce progressive ischaemic neuropathy. Because glaucomatous neuropathy is slowly progressive and the duration of experimental observation in the present study was limited to the period necessary for development of advanced glaucoma, the experimental findings were compared with observations of slowly progressive visual impairment in an aged monkey to reveal the long-term characteristics of glaucomatous neuropathy.

\section{MATERIALS AND METHODS}

We studied 11 pairs of monkey eyes from adult Japanese macaques (Macaca fuscata) weighing $5-9 \mathrm{~kg}$ provided by neurophysiologists. All experiments were performed according to the Institutional Guidelines for Animal Handling and Experimentation of the Tokyo Metropolitan Institute of Medical Science. In the experiment, 11 eyes underwent cauterisation and 11 eyes served as control. Surgical exploration of the junction of optic nerve stalk and sclera was done by superiortemporal orbitotomy under intravenous anaesthesia. The paraoptic lateral SPCA was identified with two or three branches on the surface curving into a junctional cave. Cauterisation was done using a $2 \mathrm{~mm}$ diameter bipolar disc electrode with $2 \mathrm{~mA}$ of current placed on the bent junction (schematic view in figure $3 \mathrm{~A}$, stereoscopic view in figure $3 \mathrm{~B}$ ). The procedure was carefully designed to produce incomplete blockage of vessels and avoid necrosis of the underlying nerve. To allow progressive observation, interval to pathological examination after cauterisation varied from 2 to 56 days. Pre- and postoperative fundus photographs and images from fluorescein angiography were analysed in five eyes. Intraocular pressure was not measured due to the lack of appropriate instruments. The animals were killed and exsanguinated under deep anaesthesia. The posterior halves were sliced into $6 \mu \mathrm{m}$ thick sections for paraffin processing and $14 \mu \mathrm{m}$ thick sections for cryopreservation. The anterior trabecular meshwork and LGN were also examined for spontaneous neuropathy. One of the following methods of staining was chosen for each purpose: Gomori's aldehyde-fuchsin, Elastica van 
Gieson, silver-Luxol fast blue and Nissle. For immunohistochemistry, a primary antibody-either phosphorylated monoclonal mouse antibody (SMI-31; Sternberger Monoclonal Inc., Lutherville, Maryland, USA) or non-phosphorylated neurofilament (SMI-32), neurofilament-H (NF-H; Biomol International, LP, Plymouth Meeting, Pennsylvania, USA) for both NFs, or monoclonal rabbit anti-glial fibrillary acidic protein (anti-GFAP; Sigma-Aldrich Inc, St. Louis, Missouri, USA)—was applied to paraffin-embedded sections. The procedure was performed as previously reported ${ }^{12}$ except for substrate chromogen staining with 3,3'-diaminobenzidine-nickel chloride (DABni; SigmaAldrich Inc). Secondary antibodies of rhodamine-conjugated anti-mouse immunoglobulin G (IgG) for SMI-31 and Alexa Fluor-conjugated anti-rabbit IgG for GFAP were used for double labelling. Specificity of staining was confirmed by omitting the primary antibody. A membrane-permeable tracer (Cell Tracker CMRA, Molecular Probes Inc, Eugene, Oregon, USA) was used to investigate blood flow in capillary and glial cell-capillary interaction in columnar spaces. The tracer (concentration of $30 \mu \mathrm{M} / 3 \mathrm{ml}$ of saline) was injected into the temporal retrobulbar space and examined 3 days later.

An aged monkey that had been kept for 25 years at our Institute had developed impaired visual task performance without other neurological disability. His eyes were examined for evidence of advanced glaucomatous neuropathy, using fundus photography and pathological analysis. Specimens were observed and photographed via light and confocal laser scanning (Nikon C1 TE-2000, Japan) microscopy.

\section{RESULTS}

The RGC axons formed axon fascicles with contiguous axons, with an almost 1-10 correspondence. These fascicles were grouped into bundles enveloped by the epineurium. The number of fibres contained in a bundle differed by retinal quadrant, that is, there were fewer in the temporal quadrant and more in the inferior quadrant (figure 1A). These bundles were arrayed
Figure 1 (A) Immunostaining of neurofilaments (NF-H; DABni). Figures are recomposed in the circle by cut ends of the retina. The magnified retina in the nasal $(\mathrm{N} ; \mathrm{a})$, inferior $(\mathrm{l} ; \mathrm{b})$, temporal $(\mathrm{T} ; \mathrm{c}$ ) and superior $(\mathrm{S}$; d) quadrants is shown. Axons of the retinal ganglion cells are grouped at their origin and form bundles enveloped by the epineurium. The bundle diameter differs by quadrant: $\mathrm{T}(\mathrm{c})<\mathrm{S}(\mathrm{d})<\mathrm{N}$ (a) $<$ I (b). (B) The difference between phosphorylated (a; SMI-31, DABni) and non-phosphorylated (b; SMI-32) neurofilaments in lamina cribrosa is unremarkable in the temporal quadrant, except for the innermost small bundles near the central vessel. The arteriole of the circle of Haller and Zinn penetrates inside (seen as a circle at the rightcentral border). The scattered dots in the columns indicate glial cells (a).
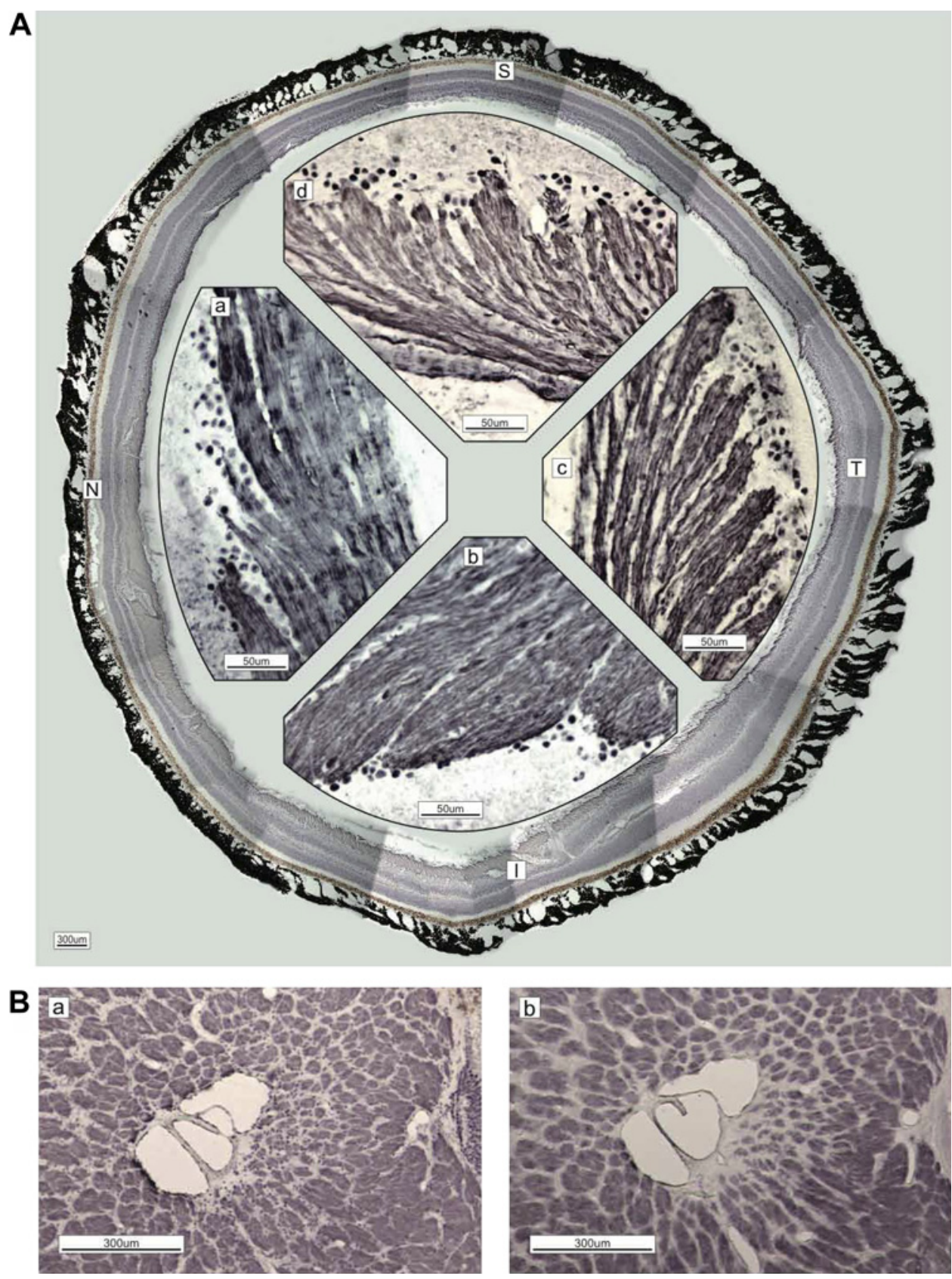
centripetally towards the optic nerve head $(\mathrm{ONH})$ along the surface in the nerve fibre layer. Bundle diameter was consistent in the prelaminar to laminar region. The macaque monkeys used in this study, the number of bundles was approximately 800 . The distribution of phosphorylated (as determined by SMI-31 staining) and non-phosphorylated (as determined by SMI-32 staining) axonal bundles did not clearly differ in the temporal optic nerve in the LC, except in the innermost small bundles temporal to the central vessel (figure $1 \mathrm{~B}$ ).

The bundles were intercalated among spaces containing capillaries, astrocytes and ECMs in columns (figure 2Aa). The astrocytes formed perpendicular lamellae and their processes were distributed around the bundles with connections to contiguous processes (figure $2 \mathrm{Ab}$ ). Arterioles in the border tissue of Elschnig penetrated the septum of the optic nerve sheath and distributed as capillaries in the intercolumnar spaces (figure $2 \mathrm{Ba}, \mathrm{b}$ ). Fluorescent tracer injected in the connective tissue of the subarachnoid space surrounding the optic nerve was found in the capillaries and septum of intercolumnar spaces (figure 2C). These uptakes represent the lack of blood-brainbarrier characteristics and non-specific permeability in the prelaminar region. In addition, astrocytes containing fluorescent particles were observed on bundles and near capillaries (figure 2D), which suggests that the fixation of astrocyte processes to unmyelinated fibres functions as metabolic bridges between capillaries and bundles. Ischaemia of the circle would affect the nerves included in this complex relationship.

Experimental cauterisation of the paraoptic branches of the lateral SPCA caused time-dependent changes in nerves in the LC At 1 week and earlier, eyes $(n=4)$ showed swelling in bundles
Figure 2 (A) Silver-Luxol fast blue staining of the frontal section of the optic nerve in the lamina cribrosa. (a) Each bundle (brown) is surrounded by a column in the lamina cribrosa septa. (b) High-magnification view of the septa shows neurofilaments (black), numerous astrocytes with processes, oligodendrocytes (oval yellowish-brown cells with nuclei) and extracellular connective tissue (light blue) circumferentially enveloping the bundle (brown). (B) Elastica van Gieson staining of the extracellular matrix of the temporal border with the subarachnoid space of the optic nerve. (a) Arterioles are visible in the surrounding connective tissue $\left({ }^{*}\right)$. A capillary (white arrowhead) is visible and elastic (red) connective tissue (black arrows) is distributed in the column. (b) Magnified view of the site of penetration of an arteriole $\left({ }^{*}\right)$ and capillary (white arrowhead) into columnar spaces (white arrow). Bundles are stained brown. (C) Membrane-permeable, thiol-binding tracer uptake (red dots) into the column (white arrows) via vessels (white arrowheads). (D) High-magnification view of capillary (white arrowheads) and astrocytes on and adjacent to nerve bundles with tracer uptake (red spots, white arrows.) The phase-differential configuration (b) shows the orientation of capillary and nerve bundles.
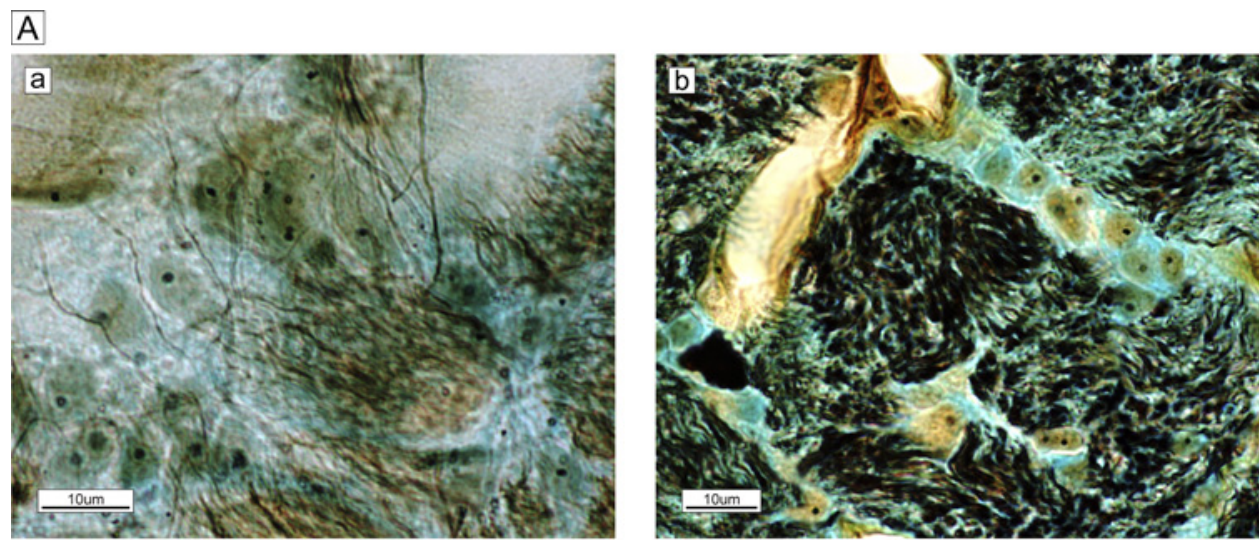

\section{B}
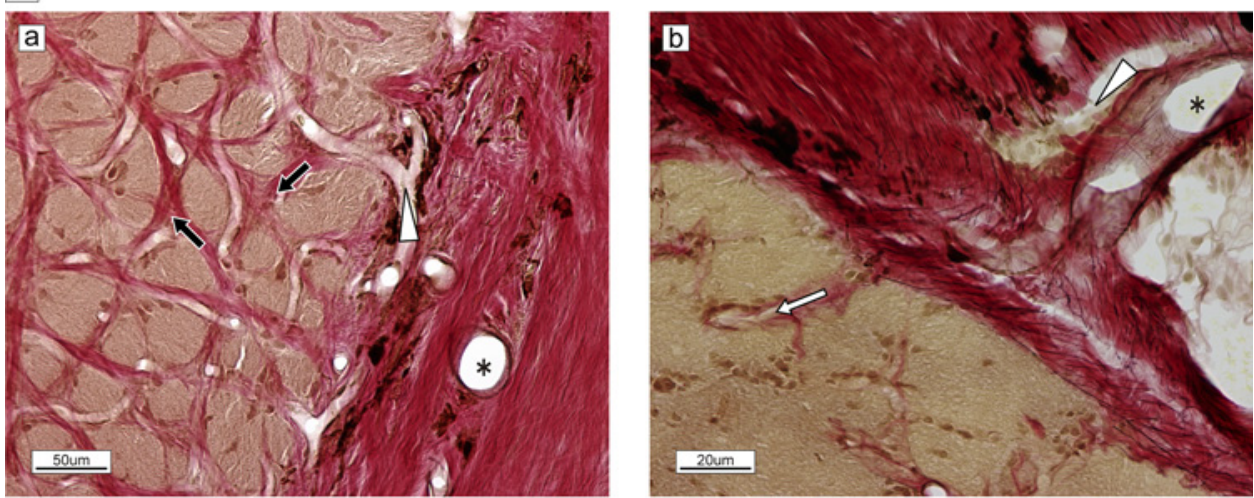

C
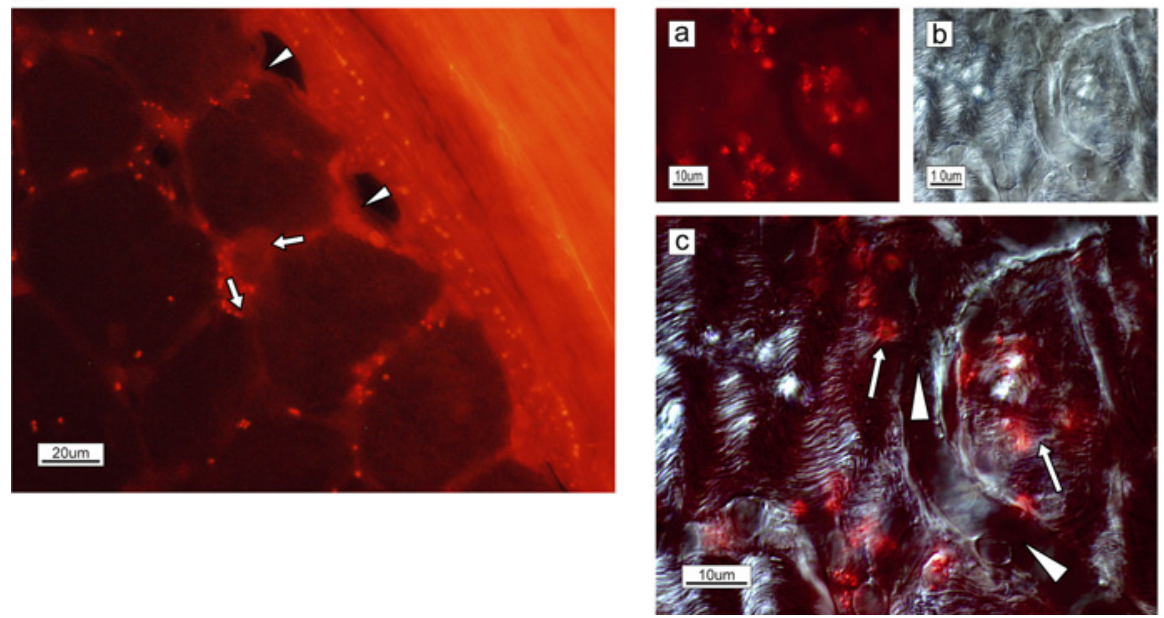
and microhaemorrhage in the LC. At three weeks $(n=3)$, we observed bundles of phosphorylated fibres with decreased immunoactivity (on SMI31 staining) in the temporal bundle (figure 3C). Double immunostaining by SMI-31 and GFAP revealed that the phosphorylated bundles were smaller (compare with figure $2 \mathrm{Ba}$ ) and that glial proliferation into the widened columns was almost entirely confined to the temporal quadrant (figure 3D). A fundus photograph showed a dark shadow in the temporal peripapillary subretina and focal round discolouration (figure $3 \mathrm{E}$ ). Fluorescein angiography revealed a filling defect in the temporal peripapillary margin (figure $3 \mathrm{~F}$ ) and focal discolouration of scar formation due to recurrent branch occlusion (white arrow in figure 3C).

These features were more obvious at five weeks $(n=5)$. Postoperative (figure 4Ab) fundus photograph revealed development of arcuate superior and inferior discolourations, whereas the papillomacular region was preserved (figure $4 \mathrm{Aa}$; preoperative image). Focal ischaemia caused the decreased immunoactivity on phosphorylated nerve bundles (stained by SMI-31, DABni) in the temporal half, except for the innermost row near the central vessel (figure 4B). A sagittal view showed a clear difference between phosphorylated (SMI-31, figure 4Ca) and non-phosphorylated (SMI-32, figure $4 \mathrm{Cb}$ ) bundles in the temporal half. Moreover, glial proliferation was clearly more extensive in the temporal than in the nasal half (GFAP, figure 4Cc). The diameter of the temporal bundles decreased, resulting in wider columnar spaces, accompanied by glial proliferation (figure 4D). Acute (2 days) deterioration of the NFs was more diffuse, probably due to focal oedema of the operated site, but it was decreased 5 days later (data not shown). These findings indicate that ischaemia in the paraoptic temporal SPCA causes progressive damage to temporal segmental optic nerve fibres. We conclude that this time-dependent deterioration corresponds to the progression of an arcuate visual field defect in glaucoma.
Figure 3 (A) Schematic view of retrobulbar structure and the vessel targeted for cauterisation (red; two branches are drawn) with a bipolar disc electrode (black stick with cord) and cautery point (black arrow). (B) Stereoscopic view of the junction between the optic nerve and sclera shows the two branches of the paraoptic lateral short posterior ciliary artery (black arrows) and the cave of the site of scleral penetration (white arrows). (C) Deterioration of temporal segmental phosphorylated axons (immunostained by SMI-31; DABni) in a sagittal section at three postoperative weeks. An artery penetrates the optic nerve from the border tissue of Elschnig at the level of the pre-lamina cribrosa (black arrow). Focal retinal discolouration (white arrows in $\mathrm{E}$ and $\mathrm{F}$ ) indicates a lesion caused by subretinal scarring of the recurrent artery (white arrow). (D) Double staining of glial fibrillary acidic protein (Alexa 488) with SMI-31 (Rhodamine 555) in the frontal section. Marked glial proliferation from the circumferential row is present in the temporal quadrant. (E) A fundus photograph shows the dark halo of a temporal disc margin (black arrowheads) with focal subretinal atrophy (white arrow). (F) Fluorescein angiography shows a filling defect in the temporal disc margin (black arrowheads) and a focal scar (white arrow) corresponding to the focal retinal lesion in $\mathrm{C}$. The macula is normal (black arrows). D, down; IOM, inferior oblique muscle; IRM, inferior rectus muscle; LRM, lateral rectus muscle; MRM, medial rectus muscle; $\mathrm{N}$, nasal; NRM, nucleus raphe magnus; $M$, the region of macula; ON, optic nerve; SOM, superior oblique muscle; SRM, superior rectus muscle, $\mathrm{T}$, temporal; $\mathrm{U}$, up.
A
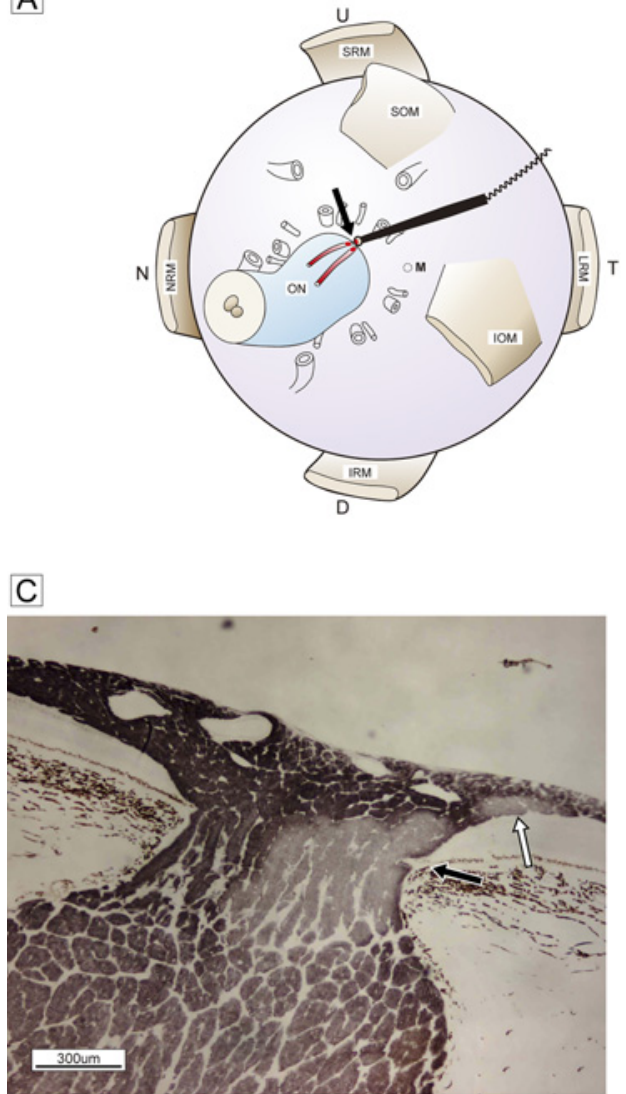

E

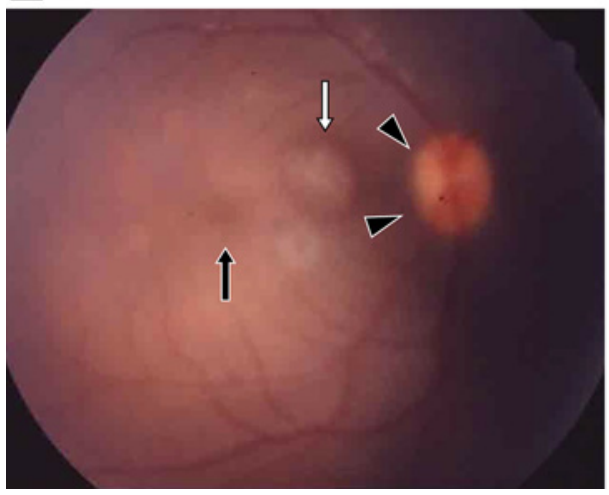

B

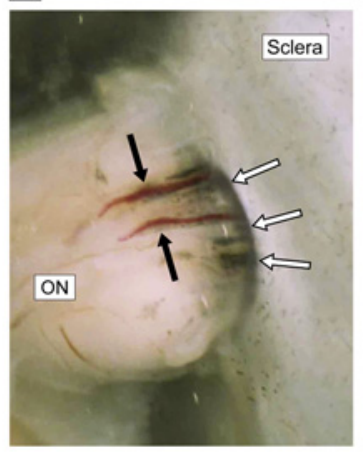

D

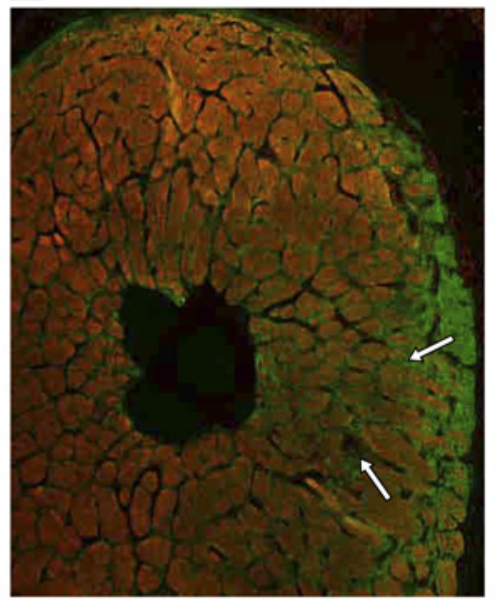

$\mathrm{F}$

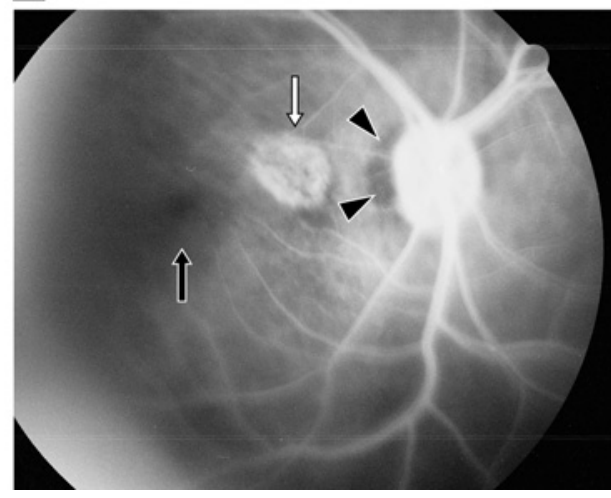


Figure 4 (A) Fundus photographs (a) before and (b) 5 weeks after surgery. After surgery, distinct subretinal arcuate discolouration is present in the superior and inferior temporal arcuate zone (white arrows) and a marginal dark halo around the disc (black arrowheads) and a normal macula (black arrow) are seen (b). Retinal vascularity is unchanged. (B) Immunostaining of phosphorylated neurofilaments in a frontal section. Diffuse deterioration of SMI-31-positive axons (DABni) is evident in the temporal $(T)$ arcuate zone, with the exception of a row in the innermost marginal bundles. (C) Immunostaining (DABni) of sagittal sections reveals a clear difference in phosphorylated (a) and non-phosphorylated (b)

neurofilament reactivity. The number of SMI-31-positive bundles decreased in the optical nerve head and lamina cribrosa in the temporal division (black arrow), in clear contrast to the nasal half (Ca). SMI-32-positive profiles in temporal bundles (black arrow) cannot be distinguished from those in nasal bundles in $\mathrm{ONH}(\mathrm{Cb})$. Expression of gliosis by glial fibrillary acidic protein increased in the temporal half (black arrow) (Cc). (D) Double staining by SMI-31 with glial fibrillary acidic protein of a frontal section demonstrates the markedly decreased diameter of phosphorylated bundles (Rhodamine 555), with widened columns and glial proliferation (Alexa 488), in the temporal arcuate zone.

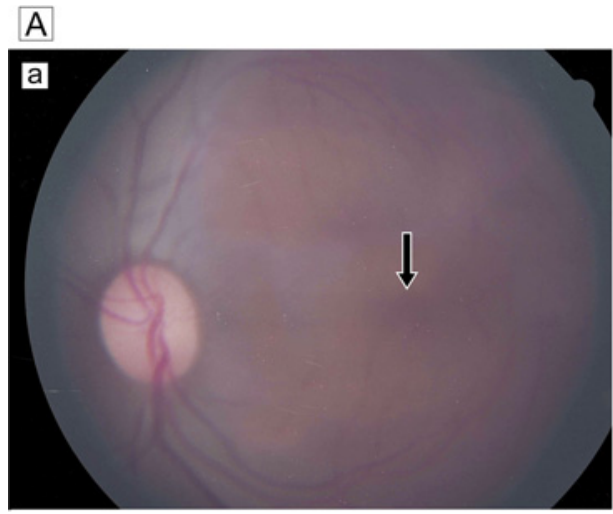

\section{B}

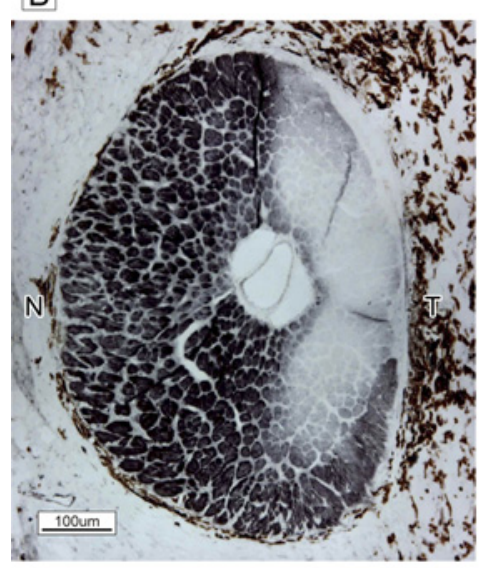

D

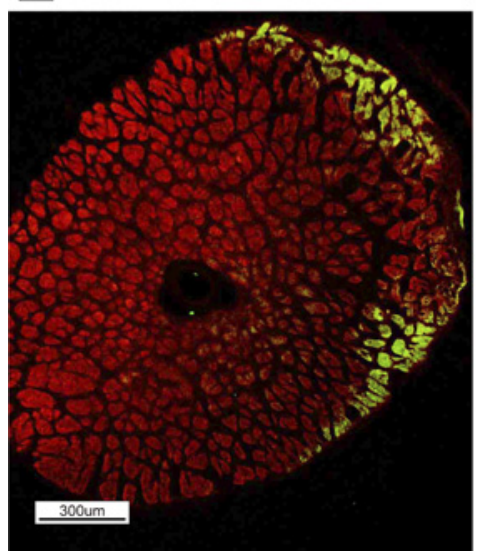

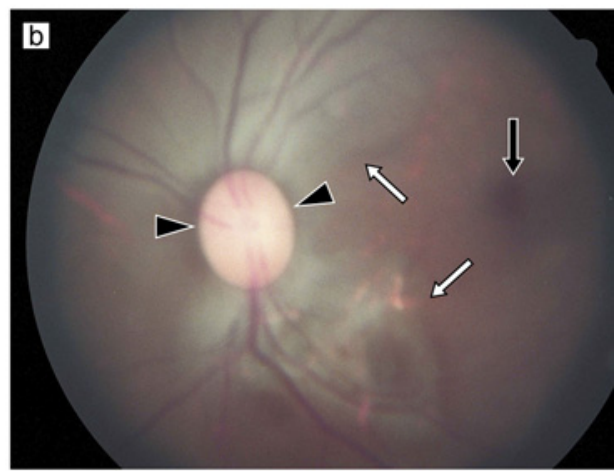
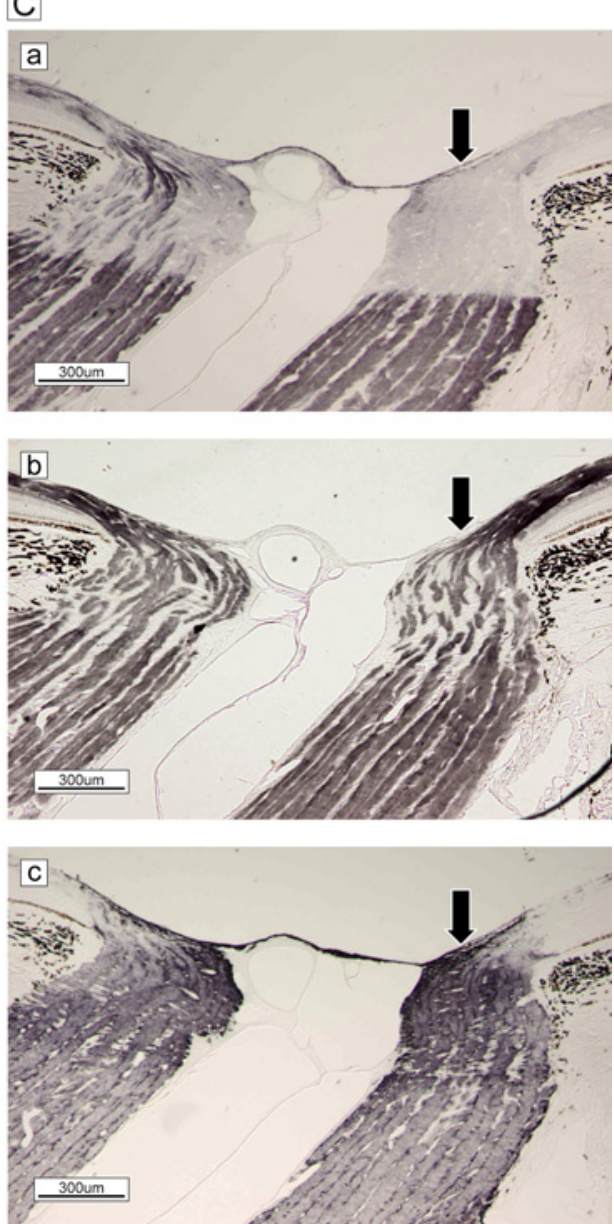

As mentioned above, we examined an aged monkey with slowly progressive visual impairment. He had no history of glaucomatous attack or pain, according to the records of the supervising veterinarian. An eye examination showed temporal pallor with excavation (figure 5C). The angle was wide open, the cornea was clear, and there was no obstruction of the trabecular meshwork (figure $5 \mathrm{~B}$ ). The LGN showed clear zonal organisation, without focal cell loss (figure $5 \mathrm{~A}$ ). The bundles of the temporal half of the optic disc were completely occupied by glial proliferation (GFAP) (figure 5D); however, bundles in the nasal half retained a clearly circumferential configuration with welldefined columnar spaces (figure 5Ea). The LC was thinner in the temporal half ( $b$ in figure $5 \mathrm{Ea}$ ) and laminar pores were irregularly deformed by ingrowth of collagenous tissues (figure $5 \mathrm{~Eb}$ ). The retinal fibre layer of the $\mathrm{ONH}$ was not atrophic, suggesting that axons from the retinal periphery were preserved. The temporal optic atrophy evident in the photograph in figure 5 is similar to the pathology in humans and confirms a diagnosis of advanced open-angle glaucoma. The observation period in the present experiment was short; thus, the unexpected observation of glaucomatous changes-although the exact duration of optic atrophy was not estimated-is highly informative in elucidating the pathological progression of this disease.

\section{DISCUSSION}

The branches of the SPCA supply blood to the region of the $\mathrm{ONH}$. There is a controversy as to whether the circle of Heller and Zinn is a complete or incomplete collateral circle between the lateral and medial paraoptic posterior ciliary arteries. ${ }^{4589} \mathrm{It}$ is generally accepted that, in nerve fibre defects in glaucoma, the 
Figure 5 Spontaneous glaucomatous optic neuropathy. (A) Nissle staining of lateral geniculate nucleus reveals a normal, layered configuration. (B) Sagittal histology of wide-open uveocorneal angle and normal trabecular meshwork stained with Gomori's aldehyde-fuchsin. (C) Fundus photograph shows the optic disc with temporal pallor and excavation and a dark halo around the temporal subretina, demonstrating a nerve fibre layer defect. The macula is normal (black arrow). (D) Double labelling of SMI-31 (green) and glial fibrillary acidic protein (red). Marked gliosis with a few bundles can be seen in the temporal half. (E) Sagittal section of optic nerve head and lamina cribrosa stained with Gomori's aldehyde-fuchsin. (a) Nerve bundles are almost completely absent and replaced by glial proliferation in the temporal half of a broad region from pre- to post-lamina cribrosa (Ea), despite a normal lateral geniculate nucleus configuration. By contrast, demarcated bundles are oriented normally in the nasal half. Superficial nerve bundles in the temporal half of the disc head are not atrophic (white arrow), presumably due to the presence of nerves from the peripheral retina. (b) High-magnification view of the boxed area in (a) reveals the details of glial proliferation (black arrow) and massive collagenous tissue (green) ingrowth (white arrow) with elastic tissue remnants of atrophied vessel wall (purple, white arrow).
A

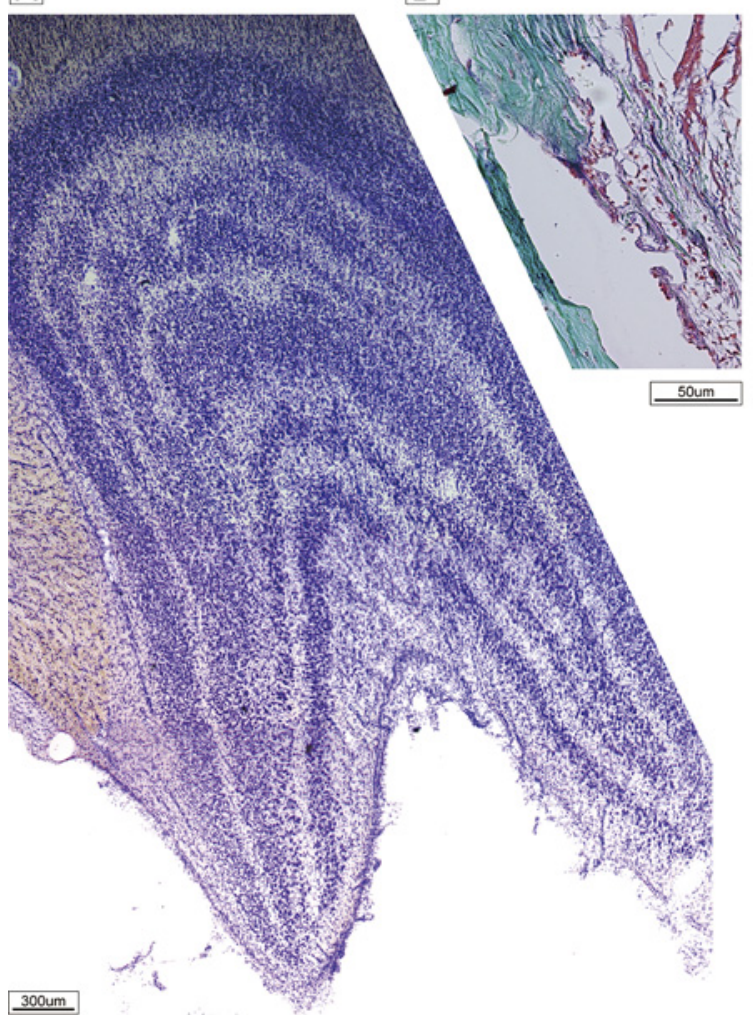

C

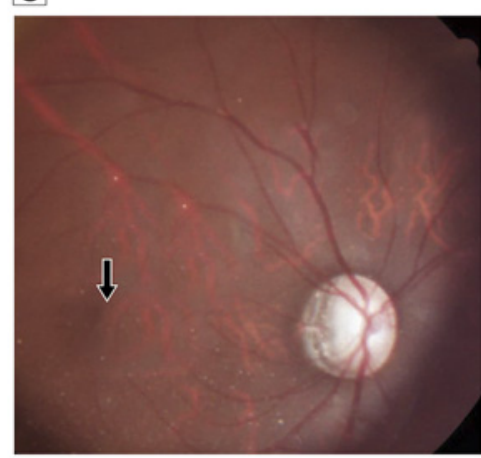

D

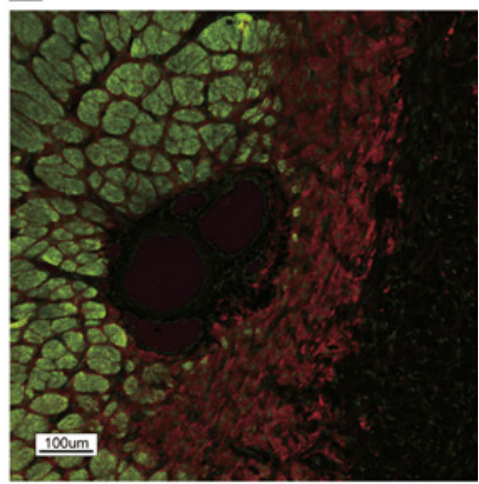

E

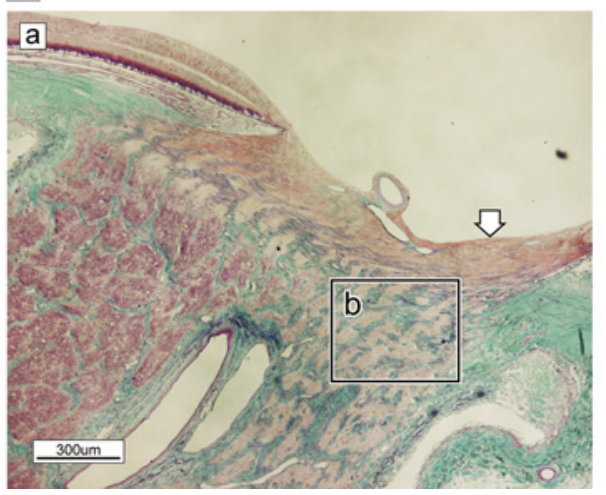

lesion site is in the disc and vascular insufficiency in $\mathrm{ONH}$ plays an important role in pathogenesis. To induce a moderate ischaemia, mechanical occlusion is more effective than injection of vasoconstrictive drugs ${ }^{6}$ or temporal posterior ciliary artery cut-off. ${ }^{10}$ In the present study, we cauterised the branches of lateral SPCA that were on the surface of entry points into the sclera. The branch of the medial SPCA was not targeted and the possibility of the results being affected is considered.

The tracing study revealed that capillaries in intercolumnar spaces are directly supplied by circumferential arterioles (figure $2 \mathrm{Da}$ ). The paraoptic branches pierced the sclero-optic junction and coursed through the border tissue of Elschnig at the level of the prelaminar cribrosa. ${ }^{13}$ The lack of blood-brain barrier properties in capillaries, ${ }^{14}$ as well as the uptake of horseradish peroxidase into the lamina via the blood stream, ${ }^{15}$ demonstrated non-specific permeability from the arterioles to the columns. The use of a membrane-permeable, thiol-reactive tracer that can be transported through the gap junction or capillary endothelium and is retained for an extended period of time in the cell body is ideal for investigating the flow connec- tion between capillaries and bundles via astrocytes. Our results clearly showed that capillaries in intercolumnar spaces are directly supplied by circumferential arterioles (figure $2 \mathrm{Da}$ ). In addition, the tracer in capillaries is transmitted to bundles via astrocyte processes, demonstrating that capillaries in the $\mathrm{LC}$ are fluid-permeable, without blood-brain barrier properties (figure $2 \mathrm{Db}$ ). This is crucial if cauterisation is to be effective in inducing selective ischaemia in the optic nerve in LC.

Glaucoma is a slowly degenerative and irreversible optic neuropathy. We attempted to study the progression of this disease by modelling the glaucoma process in macaque eyes, using an experimental ischaemic intervention in the LC. Our findings show that mechanical obstruction of circulation in the circle of Haller and Zinn causes segmental anoxia in the temporal arcuate region and progressive deterioration corresponding to that seen in glaucoma. Although a complete arterial circle, formed by both the medial and lateral SPCAs, is present in more than $75 \%$ of humans, ${ }^{5}$ our intervention was selectively applied to the temporal branches. In individuals with a complete circle, ischaemia would affect whole bundles. Nevertheless, the 
experimental results show that segmental ischaemic reactivity was restricted to the temporal arcuate region and shed light on the end-arterial features of SPCAs with sectorial blood supply.

The correlation of markers of axonal injury with the phosphorylation pattern in NFs after ischaemic intervention is crucial in characterising the extent of damage. SMI-31-labelled phosphorylated NFs and SMI-32-labelled non-phosphorylated NFs are used as markers, as their concurrent activation confirms progressive deterioration. ${ }^{16}$ We applied this method to nonmyelinated axons of the optic nerve in the LC and the findings were useful in understanding ischaemic changes. The subset of axons positively labelled for both NFs is normal in the optic nerve of control eyes (figure $1 \mathrm{Ba}, \mathrm{b}$ ); however, ischaemia resulted in a decrease in phosphorylated NFs and an increase in nonphosphorylated NFs (figure 4C). The observation period was limited in our study; however, the results suggest that functional changes related to the duration of ischaemia and the different response of the NFs precede glial proliferation in injured axons. In an advanced stage of degeneration, the absence of both SMI-31- and SMI-32-labelled NFs is evidence of functional astrocyte reactivation, resulting in GFAP production, glial scar formation and mechanical deformation of ECMs (figure 5). Thus, reactivity of NF-labelling is well correlated with axonal degeneration, and labelling profiles clearly represent functional changes due to ischaemic intervention.

Axonal injury was limited to the temporal arcuate region and our observations closely correspond to those in humans ${ }^{17}$ and macaques ${ }^{12}$ with chronic high-intraocular pressure glaucoma. Although the observation period in the present experiment was far too short to reproduce the long process of clinical development of glaucoma and our research was restricted to macaques, our findings suggest that ischaemia of paraoptic lateral SPCA is associated with the pathogenesis of glaucomatous optic neuropathy.

A study using radioactive labelling showed a greater decrease in magnocellular layers of the dorsal LGN in chronic highintraocular pressure glaucoma due to severe RGC loss, ${ }^{18}$ whereas our model of spontaneous neuropathy did not affect normal structural configuration in those layers. We cannot explain these differing findings at this time.

Interindividual variation in the vascular organisation of the LC might increase pathogenetic vulnerability in some individuals. Optic nerve damage in individuals with high myopia might be caused by an incomplete or non-anastomotic defect in the circle of Haller and Zinn. ${ }^{9}$ In addition, it is possible that mechanical obstruction of supplying vessels in the narrower temporal retrobulbar bent angle of the LC region (especially in eyes with a long axial length) could increase susceptibility to neuropathy in high myopia; however, further study is necessary to elucidate the functional importance of these anatomical variations.

The present monkey model of the degenerative process mimicking open-angle glaucoma confirmed that the mechanical and metabolic properties of circulation via the circle of Haller and Zinn play essential roles in the pathogenesis of nerve damage in glaucoma, which suggests that therapy with antiischaemic and/or vasodilative topical and/or systemic drugs could increase the clinical effectiveness of existing treatment regimens.

Acknowledgements We thank Teiko Kuroda, Michiko Imanishi and Hiroko Ueda for their excellent technical assistance in preparing the histology specimens. We also thank Dr Haruo Okado, director of the division, for his help in conducting the experiments.

Funding This work is supported by the Tokyo Metropolitan Institute Research Project for Medical Sciences.

\section{Competing interests None.}

Contributors $\mathrm{MH}$ : full responsibility for this work. Kl: responsible for animal experimental design, acquisition of data and histological procedures. TN: responsible for brain pathology with optic nerve degeneration, its analysis and interpretation of data. MT: final approval of the version to be published.

Provenance and peer review Not commissioned; externally peer reviewed.

\section{REFERENCES}

1. Hernandez MR, Neufeld AH. 35. Pathogenesis of glaucomatous optic neuropathy In: Hof PR, Mobbs CV, eds. Functional Neurobiology of Aging. San Diego: Academic Press, 2001:499-515.

2. Elkington AR, Inman CB, Steart PV, et al. The structure of the lamina cribrosa of the human eye: an immunocytochemical and electron microscopical study. Eye (Lond) 1990;4:42-57.

3. Fukuchi T, Sawaguchi S, Hara H, et al. Extracellular matrix changes of the optic nerve lamina cribrosa in monkey eyes with experimentally chronic glaucoma. Graefe's Arch Clin Exp Ophthalmol 1992:230:421-7.

4. Olver JM, Spalton DJ, McCartney AC. Microvascular study of the retrolaminar optic nerve in man: the possible significance in anterior ischaemic optic neuropathy. Eye (Lond) 1990;4:7-24.

5. Olver JM, Spalton DJ, McCartney AC. Quantitative morphology of human retrolaminar optic nerve vasculature. Invest Ophthalmol Vis Sci 1994;35:3858-66.

6. Cioffi GA, van Buskirk EM. Microvasculature of the anterior optic nerve. Surv Ophthalmol 1994;38:S107-16.

7. Onda E, Cioffi GA, Bacon DR, et al. Microvasculature of the human optic nerve. Am J Ophthalmol 1995;120:92-102.

8. Hayreh SS. Blood supply of the optic nerve head and its role in optic atrophy, glaucoma, and oedema of the optic disc. Br J Ophthalmol 1969;53:721-48.

9. Ohno-Matsui K, Futagami S, Yamashita S, et al. Zinn-Haller arterial ring observed by ICG angiography in high myopia. Br J Ophthalmol 1998;82:1357-62.

10. Itotagawa S. Observation on the choroidal vasculature. Part 3. Recovery of blood flow in choroidal circulation after experimental occlusion of the posterior ciliary arteries (In Japanese). Nippon Ganka Gakkai Zasshi 1979:83:2132-41.

11. Radius RL. Optic nerve fast axonal transport abnormalities in primates. Occurrence after short posterior ciliary artery occlusion. Arch Ophthalmol 1980;98:2018-22.

12. Kashiwagi K, Ou B, Nakamura S, et al. Increase in dephosphorylation of the heavy neurofilament subunit in the monkey chronic glaucoma model. Invest Ophthalmol Vis Sci 2003:44:154-9.

13. Lieberman MF, Maumenee AE, Green WR. Histologic studies of the vasculature of the anterior optic nerve. Am J Ophthalmol 1976;82:405-23.

14. Hofman $\mathbf{P}$, Hoyng $P$, vanderWerf $F$, et al. Lack of blood-brain barrier properties in microvessels of the prelaminar optic nerve head. Invest Ophthalmol Vis Sci 2001;42:895-901.

15. Tso MO, Shih CY, McLean IW. Is there a blood-brain barrier at the optic nerve head? Arch Ophthalmol 1975;93:815-25.

16. Herrero-Herranz $\mathbf{E}$, Pardo LA, Gold R, et al. Pattern of axonal injury in murine myelin oligodendrocyte glycoprotein induced experimental autoimmune encephalomyelitis: implications for multiple sclerosis. Neurobiol Dis 2008;30:162-73.

17. Quigley HA, Addicks EM, Green WR, et al. Optic nerve damage in human glaucoma. II. The site of injury and susceptibility to damage. Arch Ophthalmol 1981;99:635-49.

18. Dandona L, Hendrickson A, Quigley HA. Selective effects of experimental glaucoma on axonal transport by retinal ganglion cells to the dorsal lateral geniculate nucleus. Invest Ophthalmol Vis Sci 1991;32:1593-9. 